\title{
Degenerate Flag Varieties of Type A and C are Schubert Varieties
}

\section{Giovanni Cerulli Irelli ${ }^{1}$ and Martina Lanini ${ }^{2}$}

${ }^{1}$ Dipartimento di Matematica, Sapienza-Università di Roma, Piazzale Aldo Moro 5, 00185 Rome, Italy and ${ }^{2}$ Department Mathematik, Universität Erlangen-Nürnberg, Cauerstraße 11, 91058 Erlangen, Germany

Correspondence to be sent to: cerulli.math@googlemail.com

We show that any type A or C degenerate flag variety is, in fact, isomorphic to a Schubert variety in an appropriate partial flag manifold.

\section{Introduction and Main Result}

Appeared for the first time in the 19th Century to encode questions in enumerative geometry, flag varieties, and their Schubert varieties had been intensively studied since then, constituting an important investigation object in topology, geometry, representation theory, and algebraic combinatorics. In the years, several variations of these varieties have been considered (affine flag and Schubert varieties, Kashiwara flag varieties, matrix Schubert varieties, toric degenerations of flags, etc.). Among them, we want to focus on a class introduced recently by Feigin [8]: the degenerate flag varieties. These are flat degenerations of (partial) flag manifolds and turned out to be very interesting from a representation theoretic and geometric point of view. For instance, they can be used to determine a $q$-character formula for characters of irreducible modules in type A [9, 10] and $C[11,13]$. As for the geometry, degenerate flag varieties share several properties 
with Schubert varieties: they are irreducible, normal locally complete intersections with terminal and rational singularities $[8,9,13]$. In this work, we show that any degenerate flag variety of type A or C not only has a lot in common with Schubert varieties, but it is actually isomorphic to a Schubert in an appropriate partial flag variety. In short:

Theorem 1.1. Degenerate flag varieties of type A and C are Schubert varieties.

This result is based on the realization of degenerate flag varieties in terms of linear algebra, which is due to Feigin in type A [7, Theorem 2.5] and to Feigin et al. in type $C$ [13, Theorem 1.1]. This description does not use any further information on the geometry of such varieties, and hence the theorem provides an independent proof of their geometric properties such as normality, irreducibility, rational singularities, cellular decomposition, which have been established in [7-9, 13] by direct analysis.

We now state the precise version of Theorem 1.1 in the case of complete flags of type A (in Section 3, we discuss the case of partial flags, while in Section 4 we discuss the symplectic case). Let $n \geq 1$ and $B \subset \mathrm{SL}_{2 n}$ be the subgroup of upper triangular matrices. For a weight $\lambda$ of $\mathrm{SL}_{2 n}$, let $P_{\lambda}$ be its stabilizer. Let $\omega_{1}, \ldots, \omega_{2 n}$ be the fundamental weights and let $P:=P_{\omega_{1}+\omega_{3}+\cdots+\omega_{2 n-1}}$ from now on. The Weyl group of $\mathrm{SL}_{2 n}$ is $\mathrm{Sym}_{2 n}$ (the symmetric group on $2 n$ letters) and $P$ corresponds to the subgroup $W_{J}$ of $S_{2 y}$ generated by the transpositions $J=\left\{(2 i, 2 i+1)_{i=1, \ldots, n-1}\right\}$. The variety $\mathrm{SL}_{2 n} / P$ is naturally identified with the set of partial flags $W_{1} \subset W_{2} \subset \cdots \subset W_{n}$ in $\mathbb{C}^{2 n}$ such that $\operatorname{dim}\left(W_{i}\right)=2 i-1$.

The subgroup $B$ acts on $\mathrm{SL}_{2 n} / P$ (by left multiplication) and its orbits give the Bruhat decomposition:

$$
\mathrm{SL}_{2 n} / P=\coprod_{\tau \in \mathrm{Sym}_{2 n}^{J}} B \tau P / P
$$

where $\operatorname{Sym}_{2 n}^{J}$ is the set of permutations $\tau$ in $\operatorname{Sym}_{2 n}$ such that $\tau(2 i)<\tau(2 i+1)$, for $i=$ $1, \ldots, n-1$. This is the set of minimal length representatives for the cosets in $\mathrm{Sym}_{2 n} / W_{J}$. For a permutation $\tau \in \mathrm{Sym}_{2 n}^{J}$, let $\mathcal{C}_{\tau}$ be the corresponding Schubert cell in $\mathrm{SL}_{2 n} / P$, that is $B \tau P / P$, and denote by $X_{\tau}=\overline{B \tau P / P}$ its closure, that is the associated Schubert variety. Then, each Schubert cell $\mathcal{C}_{\tau}$ has exactly one point which is fixed by the action of the subgroup of diagonal matrices $T \subseteq B$, namely

$$
\left\langle e_{\tau(1)}\right\rangle\left\langle\left\langle e_{\tau(1)}, e_{\tau(2)}, e_{\tau(3)}\right\rangle<\cdots<\left\langle e_{\tau(1)}, e_{\tau(2)}, e_{\tau(3)}, \ldots, e_{\tau(2 n-1)}\right\rangle\right.
$$


(For a collection of vectors $\mathbf{v}$ of a complex vector space, we always denote by $\langle\mathbf{v}\rangle$ the subspace spanned by v.) Let $\sigma=\sigma_{n} \in \mathrm{Sym}_{2 n}$ be the permutation defined as

$$
\sigma_{n}(r)= \begin{cases}k & \text { if } r=2 k, \\ n+1+k & \text { if } r=2 k+1\end{cases}
$$

For example, for $n=5$ the permutation $\sigma$ is given by

$$
\left(\begin{array}{cccccccccc}
1 & 2 & 3 & 4 & 5 & 6 & 7 & 8 & 9 & 10 \\
6 & 1 & 7 & 2 & 8 & 3 & 9 & 4 & 10 & 5
\end{array}\right)
$$

Note that $\sigma \in \operatorname{Sym}_{2 n^{\prime}}^{J}$ indeed $\sigma(2 i)=i<\sigma(2 i+1)=n+1+i$ for $1 \leq i \leq n-1$.

Let $\mathcal{F} l_{n+1}^{a}$ denote the complete degenerate flag variety associated with $\mathrm{SL}_{n+1}$ (see Section 2 for a definition of such a variety). In [3], it is shown that $\mathcal{F} l_{n+1}^{a}$ is acted upon by the maximal torus $T$ of $\mathrm{SL}_{2 n}$ (this is recalled in Section 2).

We are now ready to state the precise version of Theorem 1.1 in the case of complete flags (the general result for partial flags is Theorem 3.1).

Theorem 1.2. There exists a $T$-equivariant isomorphism of projective varieties

$$
\zeta: \quad \mathcal{F} l_{n+1}^{a} \stackrel{\simeq}{\longrightarrow} X_{\sigma} \subset \mathrm{SL}_{2 n} / P,
$$

where $\sigma$ is the permutation given in (1.2) and $P=P_{\omega_{1}+\omega_{3}+\cdots+\omega_{2 n-1}}$.

We note that since the isomorphism $\zeta$ is $T$-equivariant, it is possible to compute the stalks of the local $T$-equivariant intersection cohomology of $\mathcal{F} l_{n+1}^{a}$ by using the parabolic analog of Kazhdan-Lusztig polynomials, defined by Deodhar [6]. This answers a question posed in [3] (and it was the original motivation for this project). Another corollary of the theorem is that the median Genocchi number $h_{n}=\chi\left(\mathcal{F} l_{n+1}^{a}\right)$ (see [7]) has another interpretation: it is the number of elements $\tau \in \operatorname{Sym}_{2 n}^{J}$ that are smaller than $\sigma$ in the (induced) Bruhat order.

As the anonymous referee has kindly pointed out, it would be interesting to analyze Theorem 1.1 in the light of the recent theory of Favorable Modules [12]. Moreover, She/He also asks what is the interplay between the Borel subgroup $B \subset \tilde{G}$ and the "degenerate group" $G^{a}$ (see [7]), where $\tilde{G}=\mathrm{SL}_{2 n}$ (respectively, $\tilde{G}=\mathrm{Sp}_{4 n-2}$ ) and $G=\mathrm{SL}_{n+1}$ (respectively, $\left.G=\mathrm{Sp}_{2 n}\right)$. Indeed both $G$ and $\tilde{G}$ act on the corresponding degenerate flag varieties 
(see Theorems 1.2 and 4.1 for the action of $\tilde{G}$ and $[7,13]$ for the action of $G^{a}$ ). This will be the subject of a future project.

The paper is organized as follows: in Section 2, we prove Theorem 1.2, in Section 3 we discuss its analog for partial degenerate flags and in Section 4 we prove the analogous results for type C. In the Appendix, we prove that the desingularization of the degenerate flag varieties provided in [9] coincides with a Bott-Samelson resolution of the Schubert variety $X_{\sigma}$.

\section{Proof of Theorem 1.2}

Given an integer $n \geq 1$, let $\mathcal{F} l_{n+1}^{a}$ denote the complete degenerate flag variety associated with SL $\mathrm{S}_{n+1}$. In [7, Theorem 2.5], it is proved that $\mathcal{F} l_{n+1}^{a}$ can be realized as follows: let $\left\{f_{1}, \ldots, f_{n+1}\right\}$ be an ordered basis of a complex vector space $V \simeq \mathbb{C}^{n+1}$ and let $\operatorname{pr}_{k}: V \rightarrow V$ be the linear projection along the line spanned by $f_{k}$, that is, $\operatorname{pr}_{k}\left(\sum a_{i} f_{i}\right)=\sum_{i \neq k} a_{i} f_{i}$. Then, there is an isomorphism

$$
\mathcal{F} l_{n+1}^{a} \simeq\left\{\left(V_{1}, \ldots, V_{n}\right) \in \prod_{i=1}^{n} \mathrm{Gr}_{i}(V) \mid \operatorname{pr}_{i+1}\left(V_{i}\right) \subset V_{i+1} \forall i=1, \ldots n-1\right\}
$$

For convenience of notation, up to an obvious change of basis of $V$, we prefer to realize $\mathcal{F} l_{n+1}^{a}$ as follows:

$$
\mathcal{F} l_{n+1}^{a} \simeq\left\{\left(V_{1}, \ldots, V_{n}\right) \in \prod_{i=1}^{n} \mathrm{Gr}_{i}(V) \mid \operatorname{pr}_{i}\left(V_{i}\right) \subset V_{i+1} \forall i=1, \ldots, n-1\right\} .
$$

Let $\left\{e_{1}, \ldots, e_{2 n}\right\}$ be an ordered basis of a vector space $W \simeq \mathbb{C}^{2 n}$. For any $i=$ $1,2, \ldots, n$, we consider the coordinate subspace $U_{n+i}:=\left\langle e_{1}, \ldots, e_{n+i}\right\rangle \subseteq W$ and the surjection $\pi_{i}: U_{n+i} \longrightarrow V$ defined on the basis vectors as

$$
\pi_{i}\left(e_{k}\right)= \begin{cases}0 & \text { if } 1 \leq k \leq i-1, \\ f_{k} & \text { if } i \leq k \leq n+1, \\ f_{k-n-1} & \text { if } n+2 \leq k \leq n+i,\end{cases}
$$


and extended by linearity to $U_{n+i}$. This induces a chain of embeddings of projective varieties

$$
\begin{aligned}
& \mathrm{Gr}_{i}(V) \longleftrightarrow \mathrm{Gr}_{2 i-1}\left(U_{n+i}\right) \longrightarrow \mathrm{Gr}_{2 i-1}(W), \\
& U \longmapsto \pi_{i}^{-1}(U) \longmapsto \pi_{i}^{-1}(U) .
\end{aligned}
$$

We call $\zeta_{i}: \mathrm{Gr}_{i}(V) \hookrightarrow \mathrm{Gr}_{2 i-1}(W)$ the concatenation of the above maps. We hence have a diagonal embedding

$$
\begin{aligned}
& \zeta: \prod_{i=1}^{n} \mathrm{Gr}_{i}(V) \longrightarrow \prod_{i=1}^{n} \mathrm{Gr}_{2 i-1}(W), \\
& \left(V_{1}, V_{2}, \ldots, V_{n}\right) \longmapsto\left(\zeta_{1}\left(V_{1}\right), \zeta_{2}\left(V_{2}\right), \ldots, \zeta_{n}\left(V_{n}\right)\right) .
\end{aligned}
$$

Let us show that $\zeta$ restricts to a map $\mathcal{F} l_{n+1}^{a} \rightarrow \mathrm{SL}_{2 n} / P$. We consider a point $\left(V_{1}, \ldots, V_{n}\right) \in$ $\mathcal{F} l_{n+1}^{a} \subset \prod_{i=1}^{n} \operatorname{Gr}_{i}(V)$; thus, $\operatorname{pr}_{i}\left(V_{i}\right) \subset V_{i+1}$ for any $i=1, \ldots, n-1$. We note that $\pi_{i+1}$ coincides with $\mathrm{pr}_{i} \circ \pi_{i}$ on $U_{n+i} \subset U_{n+i+1}$. Denoting by $W_{i}:=\zeta_{i}\left(V_{i}\right)$, we obtain

$$
W_{i} \subseteq \pi_{i+1}^{-1} \pi_{i+1}\left(W_{i}\right)=\pi_{i+1}^{-1} \operatorname{pr}_{i} \pi_{i}\left(W_{i}\right)=\pi_{i+1}^{-1} \operatorname{pr}_{i}\left(V_{i}\right) \subseteq \pi_{i+1}^{-1}\left(V_{i+1}\right)=W_{i+1} .
$$

Therefore, $\zeta$ restricts to an embedding $\zeta: \mathcal{F} l_{n+1}^{a} \hookrightarrow \mathrm{SL}_{2 n} / P$.

We now recall the action of the maximal torus $T \subset \mathrm{SL}_{2 n}$ on $\mathcal{F} l_{n+1}^{a}$ defined in [2, Section 3.1]. Let $T_{0}$ be a maximal torus of $\mathrm{GL}_{n+1}(\mathbb{C})$. Up to a change of basis, we assume that $T_{0}$ acts on $V$ by rescaling the basis vectors $f_{i}$ 's. This induces a diagonal action of $n$ copies $T_{0}^{(1)} \times \cdots \times T_{0}^{(n)}$ of $T_{0}$ on the direct sum $V^{(1)} \oplus \cdots \oplus V^{(n)}$ of $n$ copies of $V$. More precisely, we endow every copy $V^{(i)}$ with a basis $\left\{f_{1}^{(i)}, \ldots, f_{n+1}^{(i)}\right\}$ and the torus acts by rescaling the $f_{k}^{(i)}$ 's. We consider the linear map $\mathrm{pr}_{i}: V^{(i)} \rightarrow V^{(i+1)}$ defined on the basis vectors by sending $f_{k}^{(i)}$ to $f_{k}^{(i+1)}$ for $k \neq i$, and $f_{i}^{(i)}$ to zero, and extended by linearity. We define $T_{1} \subset \prod_{i=1}^{n} T_{0}^{(i)}$ to be the maximal subgroup such that each projection $\operatorname{pr}_{i}: V^{(i)} \rightarrow$ $V^{(i+1)}$ is $T_{1}$-equivariant. It can be checked that $T_{1}$ has dimension $2 n$ and hence $T_{1}$ is isomorphic to a maximal torus of $\mathrm{GL}_{2 n}(\mathbb{C})$. More explicitly, an element $\underline{\lambda}=\left(\lambda_{1}, \ldots, \lambda_{2 n}\right) \in$ $T_{1}$ acts by

$$
\underline{\lambda} \cdot f_{k}^{(i)}:= \begin{cases}\lambda_{k} f_{k}^{(i)} & \text { if } i \leq k \leq n+1, \\ \lambda_{n+1+k} f_{k}^{(i)} & \text { if } 1 \leq k \leq i-1 .\end{cases}
$$

Moreover, since the action of $T_{0}$ on $V$ induces an action on each $\operatorname{Grassmannian} \mathrm{Gr}_{i}(V)$, then the action of $T_{0}^{(1)} \times \cdots \times T_{0}^{(n)}$ on $V^{(1)} \oplus \cdots \oplus V^{(n)}$ induces an action of $T_{1}$ on the 
product of Grassmannians $\prod_{i=1}^{n} \mathrm{Gr}_{i}\left(V^{(i)}\right)=\prod_{i=1}^{n} \mathrm{Gr}_{i}(V)$. Since each projection $\mathrm{pr}_{i}$ is $T_{1^{-}}$ equivariant, this action descends to an action on $\mathcal{F} l_{n+1}^{a}$. Note that the action of a point $\underline{\lambda} \in T$ on $\mathcal{F} l_{n+1}^{a}$ coincides with the action of any of its multiples; we hence see that $T:=T_{1} \cap \mathrm{SL}_{2 n}$ also acts on $\mathcal{F} l_{n+1}^{a}$.

We now prove that the map $\zeta: \mathcal{F} l_{n+1}^{a} \hookrightarrow \mathrm{SL}_{2 n} / P$ is $T$-equivariant. The maximal torus $T$ in $\mathrm{SL}_{2 n}$ acts on $W$ (and hence on each Grassmannian $G_{k}(W)$ ) by rescaling the basis vectors $e_{k}$ 's : given $\underline{\lambda}=\left(\lambda_{1}, \ldots, \lambda_{2 n}\right) \in T$

$$
\underline{\lambda} e_{k}:=\lambda_{k} e_{k}
$$

From (2.4) and (2.5), it follows that each map $\pi_{i}$ is $T$-equivariant and hence each $\zeta_{i}$ is $T$-equivariant and hence $\zeta$ itself is $T$-equivariant.

We now describe the image $\zeta\left(\mathcal{F} l_{n+1}^{a}\right) \simeq \mathcal{F} l_{n+1}^{a}$. We claim that it is given by

$$
Y_{n}:=\left\{W_{1} \subset W_{2} \subset \cdots \subset W_{n} \mid \begin{array}{l}
\bullet \operatorname{dim} W_{i}=2 i-1 \\
\bullet\left\langle e_{1}, e_{2}, \ldots, e_{i-1}\right\rangle \subset W_{i} \\
\bullet W_{i} \subset\left\langle e_{1}, \ldots, e_{n+i}\right\rangle
\end{array}\right\} \subset \mathrm{SL}_{2 n} / P
$$

Indeed, $\zeta\left(\mathcal{F} l_{n+1}^{a}\right)$ is clearly contained in $Y_{n}$; viceversa, given a flag $W_{\bullet}:=\left(W_{1} \subset W_{2} \subset\right.$ $\left.\cdots \subset W_{n}\right)$ in $Y_{n}$, then by definition ker $\pi_{i} \subset W_{i} \subset U_{n+i}$ and hence $W_{i}=\pi_{i}^{-1}\left(\pi_{i}\left(W_{i}\right)\right)=$ $\zeta_{i}\left(\pi_{i}\left(W_{i}\right)\right)$. It follows that $W_{\bullet}=\zeta\left(\left(\pi_{1}\left(W_{1}\right), \ldots, \pi_{n}\left(W_{n}\right)\right)\right) \in \operatorname{Im} \zeta$. It remains to show that $\left(\pi_{1}\left(W_{1}\right), \ldots, \pi_{n}\left(W_{n}\right)\right) \in \mathcal{F} l_{n+1}^{a}$. This is immediately verified as follows: $\operatorname{pr}_{i}\left(\pi_{i}\left(W_{i}\right)\right)=$ $\pi_{i+1}\left(W_{i}\right) \subseteq \pi_{i+1}\left(W_{i+1}\right)$, for any $i=1, \ldots, n-1$.

To show that $Y_{n} \cong X_{\sigma}$, we observe that for any $i=1, \ldots, n$ we have

$$
\#\{l \leq 2 i-1 \mid \sigma(l) \leq k\}= \begin{cases}k & \text { if } 1 \leq k \leq i-1, \\ i-1 & \text { if } i-1 \leq k \leq n, \\ i-1+k-n & \text { if } n+1 \leq k \leq n+i, \\ 2 i-1 & \text { if } n+i \leq k \leq 2 n .\end{cases}
$$

It follows that for a partial flag $W_{\bullet} \in \mathrm{SL}_{2 n} / P$, condition $\left\langle e_{1}, e_{2} \ldots, e_{i-1}\right\rangle \subseteq W_{i} \subseteq$ $\left\langle e_{1}, e_{2} \ldots, e_{n+i}\right\rangle$ is equivalent to

$$
\operatorname{dim}\left(W_{i} \cap\left\langle e_{1}, e_{2}, \ldots, e_{k}\right\rangle\right) \geq \#\{l \leq 2 i-1 \mid \sigma(l) \leq k\}
$$


for any $i=1, \ldots, n$ and $k=1, \ldots, 2 n$. By [? , Corollary of the proof of Proposition 7, Section 10.5], $X_{\sigma}$ is precisely the locus of partial flags in $\mathrm{SL}_{2 n} / P$ satisfying (2.7). This concludes the proof of Theorem 1.2.

Remark 2.1. Theorem 1.2 and its proof have a nice and clean interpretation in terms of quivers, in the spirit of [2-4].

\section{Parabolic Case}

In this section, we discuss the parabolic analog of Theorem 1.2. Recall the vector space $V \simeq \mathbb{C}^{n+1}$ with basis $\left\{f_{1}, \ldots, f_{n+1}\right\}$ and let $\mathbf{d}=\left(d_{i}\right)_{i=1}^{s}$ be a collection of positive integers $1 \leq d_{1}<d_{2}<\cdots<d_{s} \leq n$. For any pair of indices $1 \leq i<j \leq n$, we consider the linear map $\mathrm{pr}_{i, j}: V \rightarrow V$ defined by $\mathrm{pr}_{i, j}=\mathrm{pr}_{j-1} \circ \cdots \circ \mathrm{pr}_{i+1} \circ \mathrm{pr}_{i}$, where $\mathrm{pr}_{i}$ is the projection along $f_{i}$ as before. Then, following [7, Theorem 2.5], the partial degenerate flag variety $\mathcal{F} l_{\mathbf{d}}^{a}$ is given by

$$
\mathcal{F} l_{\mathrm{d}}^{a} \simeq\left\{\left(V_{1}, \ldots, V_{s}\right) \in \prod_{l=1}^{s} \mathrm{Gr}_{d_{l}}(V) \mid \operatorname{pr}_{d_{l}, d_{l+1}}\left(V_{l}\right) \subset V_{l+1}\right\}
$$

The maximal torus $T \subset \mathrm{SL}_{2 n}$ acts on $\mathcal{F} l_{\mathrm{d}}^{a}$, in a similar way as for complete flags (see [2]). Let $\lambda:=\omega_{2 d_{1}-1}+\omega_{2 d_{2}-1}+\cdots+\omega_{2 d_{s}-1}$ and let $P=P_{\lambda}$ be the corresponding parabolic subgroup in $\mathrm{SL}_{2 n}$. The variety $\mathrm{SL}_{2 n} / P$ is naturally identified with the variety of partial flags $W_{1} \subset \cdots \subset W_{s} \subset W$ such that $\operatorname{dim} W_{i}=2 d_{i}-1(i=1,2, \ldots, s)$. We introduce the sets $K:=\{1,2, \ldots, 2 n\} \backslash\left\{2 d_{i}-1 \mid i=1,2, \ldots, s\right\}, J:=\{(k, k+1) \mid k \in K\}$, and the subgroup $W_{J}$ of Sym $_{2 n}$ generated by $J$. We have the Bruhat decomposition

$$
\mathrm{SL}_{2 n} / P \simeq \coprod_{\tau} B \tau P / P
$$

where this time $\tau$ runs over the set of minimal length representatives for the cosets in Sym $_{2 n} / W_{J}$. This set corresponds to the permutations $\tau \in \operatorname{Sym}_{2 n}$ such that $\tau\left(2 d_{i}\right)<\tau\left(2 d_{i}+\right.$ $1)<\cdots<\tau\left(2 d_{i+1}-1\right)$. We denote by $X_{\tau}=\overline{B \tau P / P}$ the corresponding Schubert variety. Let $\sigma_{\mathrm{d}}$ be the minimal length representative of the coset $\sigma_{n} W_{J} \in \operatorname{Sym}_{2 n} / W_{J}\left(\sigma_{n}\right.$ is defined in (1.2)); explicitly, $\sigma_{\mathrm{d}}$ is given by

$$
\sigma_{\mathbf{d}}(k)= \begin{cases}k-d_{i} & \text { if } k \in\left\{2 d_{i}, \ldots, d_{i}+d_{i+1}-1\right\}, \\ n+1+k-d_{i+1} & \text { if } k \in\left\{d_{i}+d_{i+1}, \ldots, 2 d_{i+1}-1\right\},\end{cases}
$$


with the conventions $d_{0}:=0$ and $d_{s+1}:=n+1$. For example, for $n=8$ and $\mathbf{d}=(2,5,7)$, we have

$$
\sigma_{\mathrm{d}}=\left(\begin{array}{cccccccccccccccc}
1 & 2 & 3 & 4 & 5 & 6 & 7 & 8 & 9 & 10 & 11 & 12 & 13 & 14 & 15 & 16 \\
1 & 9 & 10 & 2 & 3 & 4 & 11 & 12 & 13 & 5 & 6 & 14 & 15 & 7 & 8 & 16
\end{array}\right)
$$

Note that for $\mathbf{d}=(1,2, \ldots, n)$, the permutations $\sigma_{\mathbf{d}}$ and $\sigma_{n}(1.2)$ coincide.

Theorem 3.1. There exists a $T$-equivariant isomorphism

$$
\zeta: \mathcal{F} l_{\mathrm{d}}^{a} \stackrel{\simeq}{\longrightarrow} X_{\sigma_{\mathrm{d}}} \subset \mathrm{SL}_{2 n} / P_{\lambda}
$$

Proof. Recall the vector space $W \simeq \mathbb{C}^{2 n}$ with basis $\left\{e_{1}, \ldots, e_{2 n}\right\}$ and the surjections $\pi_{i}: U_{n+i} \longrightarrow V$ defined in (2.2) for $i=1,2, \ldots, n$. The map $\zeta$ is defined by sending $\left(V_{1}, \ldots, V_{s}\right) \in \mathcal{F} l_{\mathrm{d}}^{a}$ to the tuple $\left(W_{1}, \ldots, W_{s}\right) \in \mathrm{SL}_{2 n} / P_{\lambda}$ given by $W_{i}:=\pi_{d_{i}}^{-1}\left(V_{i}\right)$. It can be checked in the same way as in Section 2 that the image of $\zeta$ consists of partial flags $W_{1} \subset W_{2} \subset \cdots \subset W_{s}$ such that $\operatorname{dim} W_{i}=2 d_{i}-1$ and $\left\langle e_{1}, e_{2}, \ldots, e_{d_{i}-1}\right\rangle \subseteq W_{i} \subseteq\left\langle e_{1}, \ldots, e_{n+d_{i}}\right\rangle$. The proof now finishes as for Theorem 1.2.

\section{Symplectic Case}

In this section, we state and prove the analog of Theorem 1.2 in the case of the symplectic group. To fix notation, we start with a brief overview about symplectic flag varieties (see, e.g., [16, Chapter 6]). We consider a positive integer $n \geq 1$ and a complex vector space $W \simeq \mathbb{C}^{2 n}$ of dimension $2 n$ with ordered basis $\left\{e_{1}, e_{2}, \ldots, e_{2 n}\right\}$. We fix the bilinear form $b_{W}[\cdot, \cdot]$ on $W$ given by the following $2 n \times 2 n$ matrix

$$
E=\left(\begin{array}{cc}
0 & J \\
-J & 0
\end{array}\right)
$$

where $J$ is $n \times n$ anti-diagonal matrix with entries $(1,1, \ldots, 1)$, as usual. In particular, the form is nondegenerate and skew-symmetric. Moreover, $e_{k}^{*}=e_{2 n+1-k}$, for $k=1, \ldots, 2 n$. The group $\mathrm{Sp}_{2 n}$ consists of those matrices $A$ in $\mathrm{SL}_{2 n}$ which leave invariant the given form, that is, $b_{W}[A v, A w]=b_{W}[v, w]$ for every $v, w \in W$. More explicitly, we consider the involution $\iota: \mathrm{SL}_{2 n} \rightarrow \mathrm{SL}_{2 n}$ which sends a matrix $A$ to the matrix $E\left({ }^{t} A\right)^{-1} E^{-1}$; then the group $\mathrm{Sp}_{2 n}$ consists of $\iota$-invariant matrices. The advantage of choosing the form as above is 
that the intersection $B \cap \mathrm{Sp}_{2 n}=B^{\iota} \subset \mathrm{SL}_{2 n}$ consisting of $\iota$-fixed upper triangular matrices, is indeed a Borel subgroup of $\mathrm{Sp}_{2 n}$ whose maximal torus is precisely the subgroup $T^{\imath}=T \cap \mathrm{Sp}_{2 n}$ of $\iota$-invariant diagonal matrices.

The parabolic subgroup $P=P_{\omega_{1}+\cdots+\omega_{2 n-1}}$ of $\mathrm{SL}_{2 n}$ considered in Section 1 is mapped into itself by $\iota$ and the group of fixed points $Q:=P^{\iota}=P \cap \mathrm{Sp}_{2 n}$ is a parabolic subgroup of $\mathrm{Sp}_{2 n}$. The projective variety $\mathrm{Sp}_{2 n} / Q$ can be described as follows: for a subspace $U \in$ $\mathrm{Gr}_{k}(W)$ we denote by $U^{\perp} \in \mathrm{Gr}_{2 n-k}(W)$ the orthogonal space of $U$ in $W$. The map

$$
\iota_{k}: \mathrm{Gr}_{k}(W) \rightarrow \mathrm{Gr}_{2 n-k}(W): U \mapsto U^{\perp}
$$

is an isomorphism of projective varieties. The variety $\mathrm{SL}_{2 n} / P$ sits inside the product $\prod_{i=1}^{n} \mathrm{Gr}_{2 i-1}(W)$ and we consider the involution (still denoted by $\left.\iota\right)$

$$
\iota:=\prod_{i=1}^{n} \iota_{2 i-1}: \prod_{i=1}^{n} \mathrm{Gr}_{2 i-1}(W) \rightarrow \prod_{i=1}^{n} \mathrm{Gr}_{2 i-1}(W) .
$$

The involution $\iota$ restricts to an involution on $\mathrm{SL}_{2 n} / P$ and the variety $\mathrm{Sp}_{2 n} / Q=\left(\mathrm{SL}_{2 n} / P\right)^{\iota}$ consists of $\iota$-invariant flags.

Moreover, the involution $\iota$ (on $\mathrm{SL}_{2 n}$ ) induces an involution on the symmetric group $\operatorname{Sym}_{2 n}$ as follows: it sends $\tau \mapsto \iota(\tau)$, where $\iota(\tau)(r):=2 n+1-\tau(2 n+1-r)$, for $r=1, \ldots, 2 n$. The Weyl group of $\operatorname{Sp}_{2 n}$ coincides with the subgroup $\operatorname{Sym}_{2 n}^{\iota}$ of $\iota$-fixed elements. The element $\sigma_{n} \in \operatorname{Sym}_{2 n}$ defined in (1.2) is easily seen to be fixed by $\iota$ and it hence belongs to the Weyl group of $\mathrm{Sp}_{2 n}$. The left action of $B^{\imath}$ on $\mathrm{Sp}_{2 n} / Q$ induces the Bruhat decomposition:

$$
\mathrm{Sp}_{2 n} / Q=\coprod_{\tau \in\left(\mathrm{SYm}_{2 n}^{J}\right)^{l}} B^{i} \tau Q / Q
$$

Each Schubert cell $B^{\imath} \tau Q / Q$ coincides with the set of $\iota$-fixed points $\mathcal{C}_{\tau}^{\iota}$ of the Schubert cell $\mathcal{C}_{\tau}$ of $\mathrm{SL}_{2 n}$ and the same holds for each Schubert variety, $Z_{\tau}=\overline{B^{\iota} \tau Q / Q}=X_{\tau}^{\iota}$ (cf. [16, Proposition 6.1.1.2]).

We now state the analog of Theorem 1.2 in type C. We denote by $\operatorname{Sp}_{\mathcal{F}} l_{2 m}^{a}$ the complete degenerate flag variety associated with $\mathrm{Sp}_{2 m}$ (see below for a definition).

Theorem 4.1. There exists a $T^{l}$-equivariant isomorphism of projective varieties

$$
\zeta: \quad \quad \operatorname{Sp} \mathcal{F} l_{2 m}^{a} \stackrel{\simeq}{\longrightarrow} X_{\sigma_{n}}^{\iota} \subset \mathrm{Sp}_{2 n} / Q
$$

where $n:=2 m-1, \sigma_{n}$ is the permutation given in (1.2) and $Q=P^{\imath}$ as above. 
In Section 4.1, we prove Theorem 4.1 and in Section 4.2 we state and prove its parabolic analog.

\subsection{Proof of Theorem 4.1}

Fix an integer $m \geq 1$, a complex vector space $V$ of dimension $2 m$ with basis $\left\{f_{1}, \ldots, f_{2 m}\right\}$ and a nondegenerate skew-symmetric bilinear form $b_{V}[\cdot, \cdot]$ on $V$ such that

$$
f_{k}^{*}= \begin{cases}f_{2 m-1-k} & \text { if } 1 \leq k \leq 2 m-2 \\ f_{2 m} & \text { if } k=2 m-1\end{cases}
$$

so that $V=\left\langle f_{1}, \ldots, f_{m-1}, f_{m-1}^{*}, \ldots, f_{1}, f_{2 m-1}, f_{2 m-1}^{*}\right\rangle$. We define $n:=2 m-1$, so that $V$ has dimension $n+1$ as in the previous sections. The degenerate flag variety $\mathcal{F} l_{n+1}^{a}$ sits inside the product of Grassmannians $\prod_{i=1}^{n} \mathrm{Gr}_{i}(V)$. It can be checked that the map $\iota=\prod_{i} \iota_{i}$ : $\prod_{i=1}^{n} \mathrm{Gr}_{i}(V) \rightarrow \prod_{i=1}^{n} \mathrm{Gr}_{i}(V)$ (where $\iota_{i}$ is defined in (4.2)) restricts to a map from $\mathcal{F} l_{n+1}^{a}$ to itself, and the fixed points form the degenerate symplectic flag variety associated with $\mathrm{Sp}_{2 m}$ [13, Proposition 4.7], that is,

$$
\operatorname{Sp} \mathcal{F} l_{2 m}^{a}=\left(\mathcal{F} l_{n+1}^{a}\right)^{\iota}
$$

Thus, Theorem 4.1 will follow once we show that the diagram

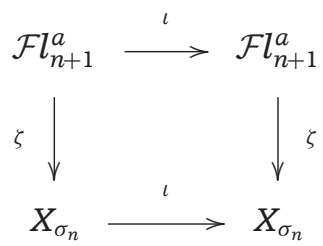

commutes, where the vertical arrows denote the T-equivariant isomorphism provided by Theorem 1.2 and the horizontal arrow in the bottom is induced by the involution (4.3). In Section 2, we proved that the isomorphism $\zeta$ is the restriction of the map $\zeta: \prod_{i=1}^{n} \mathrm{Gr}_{i}(V) \rightarrow \prod_{i=1}^{n} \mathrm{Gr}_{2 i-1}(W)$ given in (2.3). To prove (4.7), it is enough to show that 
the following diagram:

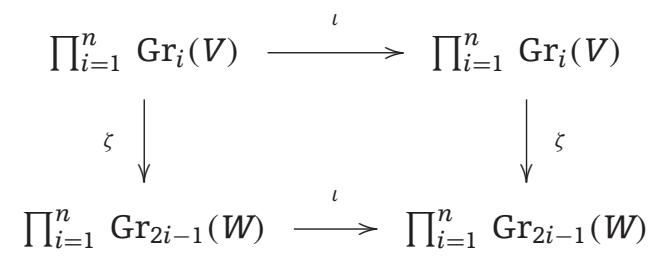

commutes. We therefore need to check that for every point $\left(V_{i}\right)_{i=1}^{n} \in \prod_{i=1}^{n} \mathrm{Gr}_{i}(V)$ and for every $i=0, \ldots, m-1$, we have

$$
\zeta_{m-i}\left(V_{m-i}\right)^{\perp}=\zeta_{m+i}\left(V_{m-i}^{\perp}\right)
$$

Recall that for every $i=1, \ldots, n, \zeta_{i}\left(V_{i}\right):=\pi_{i}^{-1}\left(V_{i}\right)$, where $\pi_{i}: U_{n+i} \rightarrow V$ is the map given in (2.2) and $U_{n+i}$ is the coordinate subspace of $W$ generated by $e_{1}, e_{2}, \ldots, e_{n+i}$. We prove the following (stronger) statement: for every $i=0, \ldots, m-1, v \in U_{n+m-i}$ and $w \in U_{n+m+i}$ we have

$$
b_{V}\left[\pi_{m-i}(v), \pi_{m+i}(w)\right]=b_{W}[v, w] .
$$

It is easy to verify that (4.10) implies (4.9): Indeed $\operatorname{dim} \zeta_{m-i}\left(V_{m-i}\right)^{\perp}=2 m+2 i-1=$ $\operatorname{dim} \zeta_{m+i}\left(V_{m-i}^{\perp}\right)$ and (4.10) implies at once that $\zeta_{m+i}\left(V_{m-i}^{\perp}\right) \subseteq \zeta_{m-i}\left(V_{m-i}\right)^{\perp}$. We will prove (4.10) by induction on $i \geq 0$. For $i=0$, we need to show that $\pi_{m}: U_{n+m} \rightarrow V$ is a map of symplectic spaces, that is, for every $v, w \in U_{n+m}$ we have $b_{V}\left[\pi_{m}(v), \pi_{m}(w)\right]=b_{W}[v, w]$. This follows easily from the definitions: Indeed, for a given $k=1, \ldots, n$, the coordinate vector subspace $U_{n+k}$ of $W$ is given by $U_{n+k}=\left\langle e_{1}, \ldots, e_{n}, e_{n}^{*}, \ldots, e_{n-k+1}^{*}\right\rangle$. In particular, $U_{n+m}$ is generated by $e_{1}, \ldots, e_{m}, \ldots, e_{n}, e_{n}^{*}, \ldots, e_{m}^{*}$ and $\pi_{m}$ is defined on the symplectic basis as follows:

$$
\pi_{m}\left(e_{k}\right)=\left\{\begin{array}{ll}
0 & \text { if } 1 \leq k \leq m-1, \\
f_{n-k}^{*} & \text { if } m \leq k \leq n-1, \\
f_{n} & \text { if } k=n,
\end{array} \quad \pi_{m}\left(e_{k}^{*}\right)= \begin{cases}f_{n-k} & \text { if } m \leq k \leq n-1, \\
f_{n}^{*} & \text { if } k=n .\end{cases}\right.
$$

We hence assume that (4.10) is true for $i \geq 0$ and we prove it for $i+1$. In view of (4.5), the map $\operatorname{pr}_{m-1+k}: V \rightarrow V(1 \leq k \leq m-1)$ is the projection along the line spanned by the basis vector $f_{m-k}^{*}$ and we denote $\operatorname{pr}_{(m-k)^{*}}:=\mathrm{pr}_{m-1+k}$. We note that the adjoint map $\operatorname{pr}_{i}^{*}$ 
of $\mathrm{pr}_{i}: V \rightarrow V$ is $\mathrm{pr}_{i^{*}}$, that is,

$$
b_{V}\left[\operatorname{pr}_{i}(v), v^{\prime}\right]=b_{V}\left[v, \operatorname{pr}_{i^{*}}\left(v^{\prime}\right)\right]
$$

for every $v, v^{\prime} \in V$. We have already observed that $\pi_{i+1}: U_{n+i+1} \rightarrow V$ restricted to $U_{n+i} \subset$ $U_{n+i+1}$ coincides with $\operatorname{pr}_{i} \circ \pi_{i}$ and, using the notation just introduced, this means that the following diagram:

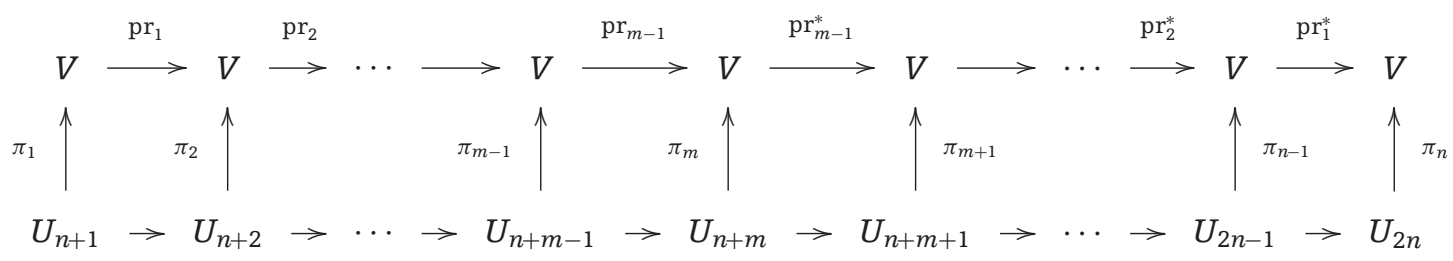

commutes (the chain of horizontal arrows in the bottom row is given by the canonical embeddings $\left.U_{n+i} \hookrightarrow U_{n+i+1}\right)$.

We can now prove (4.10). We write a nonzero element $w \in U_{n+m+(i+1)}$ as $w=$ $\mu e_{n-m-i}^{*}+w^{\prime}$ for some $w^{\prime} \in U_{n+m+i}$ and some $\mu \in \mathbb{C}$; given $v \in U_{n+m-(i+1)}$ we need to compute $b_{V}\left[\pi_{m-(i+1)}(v), \pi_{m+(i+1)}(w)\right]$. Let us first deal with the case when $w^{\prime}=0$, that is, $w=\mu e_{n-m-i}^{*}$ : we have

$$
\begin{aligned}
b_{V}\left[\pi_{m-(i+1)}(v), \pi_{m+(i+1)}(w)\right] & =\mu b_{V}\left[\pi_{m-(i+1)}(v), \pi_{m+(i+1)}\left(e_{n-m-i}^{*}\right)\right] \\
& =\mu b_{V}\left[\pi_{m-(i+1)}(v), f_{m+i}\right] \\
& =\mu b_{V}\left[\pi_{m-(i+1)}(v), f_{m-1-i}^{*}\right] .
\end{aligned}
$$

By writing $v=\sum_{k} c_{k} e_{k}$ in the symplectic basis $\left\{e_{k}\right\}$, since $\pi_{m-i-1}\left(e_{n-m-i}\right)=f_{n-m-i}=f_{m-1-i}$, we obtain

$$
b_{V}\left[\pi_{m-(i+1)}(v), \pi_{m+(i+1)}(w)\right]=\mu c_{n-m-i}=b_{W}\left[v, \mu e_{n-m-i}^{*}\right]=b_{W}[v, w] .
$$


We now consider the case when $w^{\prime} \neq 0$. In view of (4.11), (4.12), (4.13) and the induction hypothesis, we obtain

$$
\begin{aligned}
b_{V} & {\left[\pi_{m-(i+1)}(v), \pi_{m+(i+1)}(w)\right] } \\
& =b_{W}\left[v, \mu e_{n-m-i}^{*}\right]+b_{V}\left[\pi_{m-(i+1)}(v), \pi_{m+(i+1)}\left(w^{\prime}\right)\right] \\
& =b_{W}\left[v, \mu e_{n-m-i}^{*}\right]+b_{V}\left[\pi_{m-(i+1)}(v), \operatorname{pr}_{m-i-1}^{*} \circ \pi_{m+i}\left(w^{\prime}\right)\right] \\
& =b_{W}\left[v, \mu e_{n-m-i}^{*}\right]+b_{W}\left[\operatorname{pr}_{m-i-1} \circ \pi_{m-i-1}(v), \pi_{m+i}\left(w^{\prime}\right)\right] \\
& =b_{W}\left[v, \mu e_{n-m-i}^{*}\right]+b_{V}\left[\pi_{m-i}(v), \pi_{m+i}\left(w^{\prime}\right)\right] \\
& =b_{W}\left[v, \mu e_{n-m-i}^{*}\right]+b_{W}\left[v, w^{\prime}\right] \\
& =b_{W}[v, w]
\end{aligned}
$$

as desired.

\subsection{Parabolic case}

We conclude by discussing the parabolic version of Theorem 4.1, which is the type C analog of Theorem 3.1. Let $m \geq 1$ be a positive integer as in Section 4.1 , and let $\mathbf{d}=$ $\left(d_{i}\right)_{i=1}^{s}$ be a collection of positive integers $1 \leq d_{1}<d_{2}<\cdots<d_{s} \leq 2 m$ preserved by the map $d_{i} \mapsto 2 m-d_{i}$. The involution $\iota=\prod \iota_{i}: \prod_{i=1}^{s} \operatorname{Gr}_{d_{i}}(V) \rightarrow \prod_{i=1}^{s} G_{d_{i}}(V)$ is hence well-defined and restricts to a map from $\mathcal{F} l_{\mathrm{d}}^{a}$ to itself. The fixed points form the partial degenerate symplectic flag variety $\operatorname{Sp} \mathcal{F}_{\mathbf{d}}^{a}\left[13\right.$, Proposition 4.9], that is, $\operatorname{Sp} \mathcal{F}_{\mathbf{d}}^{a}=\left(\mathcal{F} l_{\mathbf{d}}^{a}\right)^{\iota}$.

Let $\lambda$ and $P_{\lambda}$ as in Section 3, so that $X_{\sigma_{\mathrm{d}}} \subset \mathrm{SL}_{2 m} / P_{\lambda}$. Let $Q:=P_{\lambda}^{\iota}$ be the parabolic subgroup of $\mathrm{Sp}_{2 m}$. The projective variety $\mathrm{Sp}_{2 m} / Q$ coincides with the $\iota$-fixed points of $\mathrm{SL}_{2 m} / P_{\lambda}$, that is, $\mathrm{Sp}_{2 m} / Q=\left(\mathrm{SL}_{2 m} / P_{\lambda}\right)^{\iota}$. Moreover, since the permutation $\sigma_{\mathrm{d}}$ is fixed by $\iota$, the corresponding Schubert variety in $\mathrm{Sp}_{2 m} / Q$ is the variety of $\iota$-fixed points $X_{\sigma_{\mathrm{d}}}$ of $X_{\sigma_{\mathrm{d}}}$. From the commutativity of Diagram (4.8), together with Theorem 3.1, we obtain the following result.

Theorem 4.2. There exists a $T^{\iota}$-equivariant isomorphism of projective varieties

$$
\zeta: \quad \quad \quad \operatorname{SpF}_{\mathrm{d}}^{a} \stackrel{\simeq}{\longrightarrow} X_{\sigma_{\mathrm{d}}}^{\iota} \subset \operatorname{Sp}_{2 n} / Q
$$

where $\sigma_{\mathbf{d}}$ is the permutation given in (3.1). 


\section{Funding}

The work of M.L. was financed by DFG SPP1388 and "Teoria delle rappresentazioni e applicazioni, Progetto di Ateneo 2012, Sapienza-Università di Roma". The work of G.C.I. was financed by the national FIRB grant RBFR12RA9W.

\section{Acknowledgements}

The main part of this project took place during M.L. stay at the Department of Mathematics "Guido Castelnuovo" of "Sapienza-Università di Roma". We thank that institution for the perfect working conditions. We thank Rocco Chirivì, Francesco Esposito, and Paolo Bravi for helpful discussions on a previous version of the present paper. We are especially grateful to Corrado De Concini, Michael Finkelberg, and Evgeny Feigin for their useful comments and to Peter Littelmann and Oksana Yakimova for several discussions about possible applications of this work.

\section{Appendix: Desingularizations}

In [9], a resolution of the singularities of a type A degenerate flag variety is constructed. In view of Theorem 1.2, it is natural to ask if such a desingularization coincides with a Bott-Samelson resolution of the corresponding Schubert variety. In this section, we show that this is indeed the case. To state and prove the result we need a combinatorial model that we discuss in Section A.1. In Section A.2, we recall the construction of Feigin and Finkelberg. In Section A.3, we construct a Bott-Samelson resolution of $X_{\sigma_{n}}$. In Section A.4, we prove that the two desingularizations coincide (see Theorem A.6). In the whole section $n \geq 1$ is a fixed integer.

\section{The quiver $\Gamma_{n}$}

We denote by $\Gamma_{n}$ the quiver (i.e., the oriented graph) with $N:=\left(\begin{array}{c}n+1 \\ 2\end{array}\right)$ vertices $\alpha_{i, j}$ with $1 \leq$ $i \leq j \leq n$ and an oriented edge $\alpha_{i, j} \rightarrow \alpha_{i+1, j}$ (for every $1 \leq i<j \leq n$ ), and an oriented edge $\alpha_{i, j} \rightarrow \alpha_{i, j+1}$ (for $1 \leq i \leq j<n$ ). The quiver $\Gamma_{n}$ is the famous Auslander-Reiten quiver of the equioriented type A quiver algebra (see, e.g., [1]). For future reference, we embed the quiver $\Gamma_{n}$ into the decorated quiver $\tilde{\Gamma}_{n}$ obtained from $\Gamma_{n}$ by adding $2 n+1$ extra vertices $\alpha_{0,1}, \alpha_{0,2}, \alpha_{0,3}, \ldots, \alpha_{0, n}, \alpha_{0, n+1}, \alpha_{1, n+1}, \alpha_{2, n+2}, \ldots, \alpha_{n, 2 n}$ and oriented edges $\alpha_{0, i} \rightarrow \alpha_{1, i}$ (for $1 \leq$ $i \leq n$ ) and $\alpha_{i, n} \rightarrow \alpha_{i, n+1}$ (for $1 \leq i \leq n$ ). For example for $n=4$, the following is $\tilde{\Gamma}_{4}$ with the 
extra vertices highlighted:

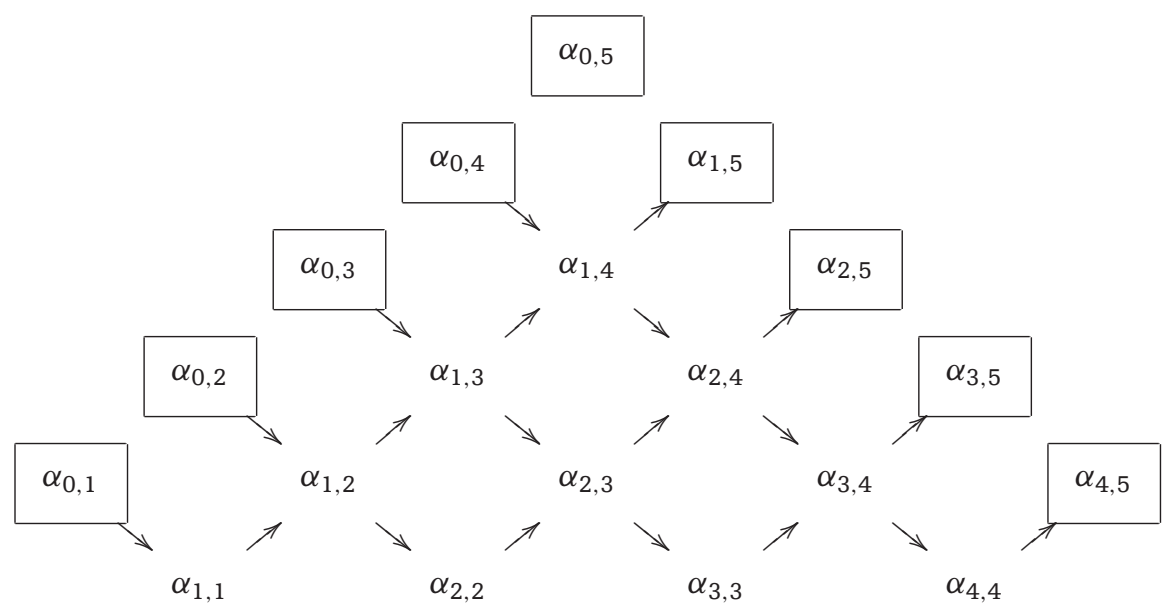

Given an index $\ell=0,1, \ldots, 2 n$, we define the $\ell$ th column of $\tilde{\Gamma}_{n}$ as the set of all vertices $\alpha_{i, j}$ such that $i+j-1=\ell$. For example, the vertex $\alpha_{i, i}$ lies on the column $2 i-1$. Similarly, given an index $r=1,2, \ldots, n+2$, we define the $r$ th row of $\tilde{\Gamma}_{n}$ as the set of vertices $\alpha_{i, j}$ of $\tilde{\Gamma}_{n}$ such that $j-i+1=r$. Note that $\Gamma_{n} \subset \tilde{\Gamma}_{n}$ has $2 n-1$ columns and $n$ rows; the row at the bottom consists of the vertices $\left\{\alpha_{i, i}\right\}_{i=1}^{n}$ and the top row consists of the single vertex $\alpha_{1, n}$. Following [9], we order the vertices of $\Gamma_{n}$ as $\beta_{1}<\beta_{2}<\cdots<\beta_{N}$ so that every row is ordered from left to right and every element of a row is smaller than every element in the rows below. More precisely, we put $\beta_{1}:=\alpha_{1, n}$, and for $\beta_{k}:=\alpha_{i_{k}, j_{k}}$ we put

$$
\beta_{k+1}:= \begin{cases}\alpha_{i_{k}+1, j_{k}+1} & \text { if } j_{k}<n, \\ \alpha_{1, n-i_{k}} & \text { if } j_{k}=n .\end{cases}
$$

To illustrate: $\beta_{1}=\alpha_{1, n}, \beta_{N}=\alpha_{n, n}$, and $\beta_{N-k}=\alpha_{n-k, n-k}$ for $k=0,1, \ldots, n-1$. We sometimes need to extend this ordering on the vertices of $\Gamma_{n}$ to an ordering on the vertices of $\tilde{\Gamma}_{n}$. We do this in the following way: we define $\beta_{0}:=\alpha_{0,1}$ and

$$
\beta_{-k}:=\alpha_{0, k+1}, \quad \beta_{-n-k}:=\alpha_{k, n+1}
$$

for every $1 \leq k \leq n$. We note that the vertex $\beta_{-m}$ lies on the $m$ th column of $\tilde{\Gamma}_{n}$ (for $0 \leq m \leq$ $2 n$ ). We always use the following notation: the vertex $\beta_{k}$ is equal to $\alpha_{i_{k}, j_{k}}$ and it lies on the column $\ell_{k}=\ell_{\beta_{k}}:=i_{k}+j_{k}-1$. Moreover, for a vertex $\beta_{k}$ of $\Gamma_{n}$ we denote by $\beta_{k}^{-}$and $\beta_{k}^{+}$the vertices of $\tilde{\Gamma}_{n}$ given by

$$
\beta_{k}^{-}:=\alpha_{i_{k}-1, j_{k}} \quad \beta_{k}^{+}:=\alpha_{i_{k}, j_{k}+1} .
$$


By construction, both $\beta_{k}^{-}$and $\beta_{k}^{+}$are strictly smaller than $\beta_{k}$ and $\ell_{\beta_{k}^{ \pm}}=\ell_{\beta_{k}} \pm 1$. We will also need the following notation: for a vertex $\beta_{k}$ of $\Gamma_{n}$ and a column index $\ell \in[1,2 n-1]$ of $\Gamma_{n}$ we define the index

$$
\left(\beta_{k}: \ell\right)=\max \left\{\beta_{s} \mid-2 n \leq s \leq k, \beta_{s} \text { lies on the } \ell \text { th column }\right\}
$$

The following equalities are a direct consequence of the definition:

$$
\begin{aligned}
\left(\beta_{k}: \ell_{k}\right) & =\beta_{k}, \\
\left(\beta_{k}: \ell_{k} \pm 1\right) & =\beta_{k}^{ \pm}, \\
\left(\alpha_{1, j}: \ell\right) & =\beta_{-\ell} \quad \forall 0 \leq \ell<j, \\
\left(\beta_{N}: 2 k-1\right) & =\beta_{N-(n-k)} \quad \forall 1 \leq k \leq n, \\
\left(\beta_{k}: t\right) & =\left(\beta_{k-1}: t\right) \quad \forall t \neq \ell_{k} .
\end{aligned}
$$

The following technical result will be used later.

Lemma .1. For $1 \leq k \leq N$ and $1 \leq t \leq 2 n-2$, either $\left(\beta_{k}: t+1\right)=\left(\beta_{k}: t\right)^{+}$or $\left(\beta_{k}: t+1\right)^{-}=$ $\left(\beta_{k}: t\right)$.

Proof. Let $\beta_{s}:=\left(\beta_{k}: t\right)$ and $\beta_{r}:=\left(\beta_{k}: t+1\right)$. Then, $\beta_{s}$ and $\beta_{r}$ lie in different columns and either $\beta_{s}<\beta_{r}$ or $\beta_{r}<\beta_{s}$. If $\beta_{s}<\beta_{r}$, then $\beta_{r}^{-}=\beta_{s}$ by maximality of $\beta_{s}$. If $\beta_{r}<\beta_{s}$, then $\beta_{r}=\beta_{s}^{+}$ by maximality of $\beta_{r}$.

\section{The desingularization of Feigin and Finkelberg}

In this section, we recall the construction of a desingularization $R_{n} \longrightarrow \mathcal{F} l_{n}^{a}$ of the complete degenerate flag variety $\mathcal{F} l_{n}^{a}$ due to Feigin and Finkelberg [9]. We fix a complex vector space $V$ of dimension $n+1$ and ordered basis $\left\{f_{1}, \ldots, f_{n+1}\right\}$. For every $1 \leq k \leq N$, we consider the coordinate subspace $V_{\beta_{k}}$ of $V$ given by

$$
V_{\beta_{k}}:=\operatorname{Im}\left(\mathrm{pr}_{j_{k}-1} \circ \cdots \circ \mathrm{pr}_{i_{k}}\right)=\left\langle f_{1}, \ldots, f_{i_{k}-1}, f_{j_{k}}, \ldots, f_{n+1}\right\rangle
$$

with the convention that $f_{0}=0$ so that $V_{1, j}=\left\langle f_{j}, \ldots, f_{n+1}\right\rangle$ (as before, the parenthesis $\langle\cdots\rangle$ denotes the C-linear span of the collection of vectors enclosed within them and $\operatorname{pr}_{k}: V \rightarrow V$ is the projection along the line generated by $f_{k}$ ). Note that the coordinate subspace $V_{\beta_{k}}$ is the analogous of $W_{i_{k}, j_{k}}$ in [9] taking into account our notation (2.1). We 
decorate the collection $\left(V_{\beta_{k}}\right)_{k=1}^{N}$ with the subspaces

$$
V_{\beta_{0}}=V_{\beta_{-k}}:=\{0\}, \quad V_{\beta_{-n-k}}:=V
$$

for every $k=1, \ldots, n$. In [9, Definition 2.1], the variety $R_{n}$ is defined as the variety of collections $\left(Z_{\beta_{k}}\right)_{k=1}^{N}$ such that

$$
\begin{aligned}
& Z_{\beta_{k}} \in \mathrm{Gr}_{i_{k}}\left(V_{\beta_{k}}\right), \\
& Z_{\beta_{k}^{-}} \subset Z_{\beta_{k}}, \\
& \operatorname{pr}_{j_{k}}\left(Z_{\beta_{k}}\right) \subset Z_{\beta_{k}^{+}},
\end{aligned}
$$

with the convention that $Z_{0, k}=\{0\}$ and $Z_{k, n+1}=V$ for $1 \leq k \leq n$. The variety $R_{n}$ is a tower of $\mathbf{P}^{1}$-fibrations: for every $1 \leq s \leq N$ we consider the variety $R_{n}(s)$ of "truncated" collections $\left(Z_{\beta_{k}}\right)_{k=1}^{S}$ satisfying conditions (.10), (.11), (.10). Then, there is a natural epimorphism

$$
p_{n}(s): \quad R_{n}(s+1) \longrightarrow R_{n}(s):\left(Z_{\beta_{i}}\right)_{1 \leq i \leq s+1} \mapsto\left(Z_{\beta_{i}}\right)_{1 \leq i \leq s}
$$

whose fiber is $\mathbb{P}^{1}$. In particular, $R_{n}$ is a smooth projective variety and the map

$$
p_{n}: R_{n} \rightarrow \mathcal{F} l_{n+1}^{a}:\left(Z_{\beta_{k}}\right)_{k=1}^{N} \mapsto\left(Z_{i, i}\right)_{1 \leq i \leq n}
$$

is a desingularization of $\mathcal{F} l_{n+1}^{a}([9])$.

\section{A Bott-Samelson resolution of $X_{\sigma_{n}}$}

In this section, we discuss a Bott-Samelson resolution of the Schubert variety $X_{\sigma_{n}}$ (see (1.2)). We choose the following reduced expression for the permutation $\sigma_{n} \in \operatorname{Sym}_{2 n}$ :

$$
\sigma_{n}=s_{n} \circ\left(s_{n-1} \circ s_{n+1}\right) \circ\left(s_{n-2} \circ s_{n} \circ s_{n+2}\right) \circ \cdots \circ\left(s_{1} \circ s_{3} \circ \cdots \circ s_{2 n-1}\right)
$$

$\left(s_{i}\right.$ denotes the simple transposition $(i, i+1)$ ). In particular, we see that the length of $\sigma_{n}$ is $N=\left(\begin{array}{c}n+1 \\ 2\end{array}\right)$ and the simple reflections appearing in (.13) are in bijection with the vertices of $\Gamma_{n}$ as follows:

$$
\sigma_{n}=\tau_{\beta_{1}} \circ \tau_{\beta_{2}} \circ \cdots \circ \tau_{\beta_{N}} .
$$

To illustrate: $\tau_{\beta_{1}}=s_{n}, \tau_{\beta_{2}}=s_{n-1}, \tau_{\beta_{N}}=s_{2 n-1}$. By construction, $\tau_{\beta_{k}}=s_{\ell_{k}}$. 
We fix a complex vector space $W$ of dimension $2 n$ with ordered basis $\left\{e_{1}, \ldots, e_{2 n}\right\}$. Given two complete flags

$$
U_{\bullet}=\left(U_{1} \subset U_{2} \subset \cdots \subset U_{2 n-1} \subset W\right), \quad W_{\bullet}=\left(W_{1} \subset W_{2} \subset \cdots \subset W_{2 n-1} \subset W\right)
$$

in $\mathrm{SL}_{2 n} / B$ and an integer $k=1,2, \ldots, 2 n-1$, the pair $\left(U_{\bullet}, W_{\bullet}\right)$ is said to be in relative posi-

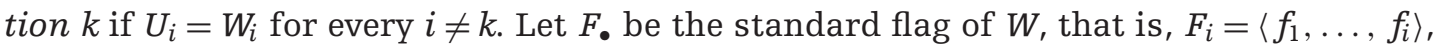
for every $0 \leq i \leq 2 n\left(F_{0}:=\{0\}\right)$. Note that the subspace $F_{n+i}$ was denoted by $U_{n+i}$ before and we freely use the two notation. We consider the variety

$$
\mathrm{BS}_{n}:=\left\{\left(U_{\bullet}^{\beta_{k}}\right)_{k=0}^{N} \in\left(\mathrm{SL}_{2 n} / B\right)^{N+1} \mid U_{\bullet}^{\beta_{0}}=F_{\bullet} \text { and }\left(U_{\bullet}^{\beta_{k+1}}, U_{\bullet}^{\beta_{k}}\right) \text { is in relative position } \ell_{k+1}\right\}
$$

This variety is the Bott-Samelson variety associated with the reduced expression (.13) of $\sigma_{n}$. In particular, the following result is well-known [5, 15].

Proposition .2. The variety $\mathrm{BS}_{n}$ is smooth. The map

$$
\rho_{n}: B S_{n} \longrightarrow X_{\sigma_{n}}:\left(U_{\bullet}^{\beta_{k}}\right)_{k=0}^{N} \longmapsto\left(U_{2 k-1}^{\beta_{N}}\right)_{k=1}^{n}
$$

is a desingularization.

We now give a description of $\mathrm{BS}_{n}$ which will be used later.

Definition .3. Let $B_{n}$ be the projective variety of collections $\left(U_{\beta_{k}}\right)_{k=-2 n}^{N}$ (indexed by the vertices of $\tilde{\Gamma}_{n}$ ) such that, for every $1 \leq k \leq N$ and $0 \leq t \leq 2 n$,

$$
\begin{aligned}
& U_{\beta_{-t}}=F_{t}, \\
& U_{\beta_{k}} \in \mathrm{Gr}_{\ell_{k}}(W), \\
& U_{\beta_{k}} \subset U_{n+i_{k}}, \\
& U_{\beta_{k}^{-}} \subset U_{\beta_{k}} \subset U_{\beta_{k}^{+}} .
\end{aligned}
$$


Proposition .4. The map $\theta_{n}: \mathrm{BS}_{n} \rightarrow B_{n}$ which sends a point $U_{\bullet}^{\beta_{\bullet}}=\left(U_{\bullet}^{\beta_{k}}\right)_{k=0}^{N}$ to the collection $\theta_{n}\left(U_{\bullet}^{\beta_{\bullet}}\right)=\left(U_{\beta_{k}}\right)_{k=-2 n}^{N}$ defined by

$$
U_{\beta_{k}}:= \begin{cases}U_{\ell_{k}}^{\beta_{k}} & \text { if } k \geq 0 \\ U_{k}^{\beta_{0}} & \text { if } k<0\end{cases}
$$

is an isomorphism of projective varieties. The inverse $\psi=\theta_{n}^{-1}$ sends a point $\left(U_{\beta_{k}}\right)_{k=-2 n}^{N}$ of $B_{n}$ to the collection of complete flags $\left(U_{\bullet}^{\beta_{k}}\right)_{k=0}^{N}$ defined by

$$
U_{t}^{\beta_{k}}:=U_{\left(\beta_{k}: t\right)}
$$

for every $1 \leq t \leq 2 n-1$.

Proof. Let us prove that the map $\theta_{n}$ is well-defined. This is based on the following technical result.

Lemma .5. Let $\left(U_{\bullet}^{\beta_{k}}\right)_{k=0}^{N}$ be a point of $\mathrm{BS}_{n}$. Then,

(i) For every $1 \leq k \leq N$, and $1 \leq t \leq 2 n-1$ we have

$$
U_{t}^{\beta_{k}}=U_{t}^{\left(\beta_{k}: t\right)}
$$

(ii) For $1 \leq k \leq N$

$$
F_{j_{k}-1} \subset U_{\ell_{k}}^{\beta_{k}} \subset F_{n+i_{k}}
$$

Proof. By definition, $U_{t}^{\beta_{k}}=U_{t}^{\beta_{k-1}}$ for $t \neq \ell_{k}$ and, in particular, (.21) holds for $k=1$. By induction on $k \geq 1$, using (.7), we obtain the desired (.21) .

If $\beta_{k}=\alpha_{1, j_{k}}$, then $\ell_{k}=j_{k}$ and $\left(\beta_{k}: j_{k}-1\right)=\beta_{-\left(j_{k}-1\right)}$ (see (.5)); in view of (.21) we have $U_{\ell_{k}-1}^{\beta_{k}}=U_{\ell_{k}-1}^{\beta_{0}}=F_{\ell_{k}-1}$ and hence $F_{j_{k}-1}=U_{\ell_{k}-1}^{\beta_{k}} \subset U_{\ell_{k}}^{\beta_{k}}$. Similarly, if $\beta_{k}=\alpha_{i, n}$, then $\ell_{k}=n+i-1$ and $\left(\beta_{k}: \ell_{k}+1\right)=\beta_{k}^{+}=\beta_{-(n+i)}$; in view of (.21), $U_{\ell_{k}+1}^{\beta_{k}}=U_{\ell_{k}+1}^{\beta_{0}}=F_{n+i}$ and hence $U_{\ell_{k}}^{\beta_{k}} \subset U_{\ell_{k}+1}^{\beta_{k}}=$ $F_{n+i}$. We hence assume that both $\beta_{k}^{-}$and $\beta_{k}^{+}$are vertices of $\Gamma_{n}$. In this case, we have

$$
U_{\ell_{k}-1}^{\beta_{k}^{-}}=U_{\ell_{k}-1}^{\beta_{k}} \subset U_{\ell_{k}}^{\beta_{k}} \subset U_{\ell_{k}+1}^{\beta_{k}}=U_{\ell_{k}+1}^{\beta_{k}^{+}} .
$$

By induction on the row index (using (.2)), the desired (.22) follows. 
Given $U_{\bullet}^{\beta} \in \mathrm{BS}_{n}$, in view of Lemma A.5 we have $U_{\ell_{k}}^{\beta_{k}} \in \mathrm{Gr}_{\ell_{k}}\left(U_{n+i_{k}}\right)$; moreover,

$$
U_{\ell_{\beta_{k}^{-}}^{\beta_{k}^{-}}}^{\beta_{-}^{-}}=U_{\ell_{k}-1}^{\beta_{k}} \subset U_{\beta_{k}} \subset U_{\ell_{k}+1}^{\beta_{k}}=U_{\ell_{\beta_{k}^{+}}^{\beta_{+}^{+}}}
$$

and hence $\theta_{n}\left(U_{\bullet}^{\beta_{\bullet}}\right)$ satisfies all the four conditions (.16)-(.19) and the map $\theta_{n}$ is welldefined and it is clearly algebraic.

With the help of Lemma A.1 and in view of (.21), one easily checks that the map $\psi$ is well-defined. It is clearly algebraic and the composition with $\theta_{n}$ is the identity.

\section{Main result}

We are now ready to state and prove the main result of the this appendix.

Theorem .6. There exists an isomorphism $\psi_{n}: R_{n} \longrightarrow \mathrm{BS}_{n}$ of projective varieties such that the following diagram:

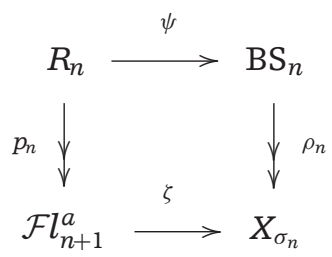

commutes (where the map $\zeta$ is the one in Theorem 1.2).

Proof. Recall that for every $1 \leq i \leq j \leq n$ we have a commutative diagram

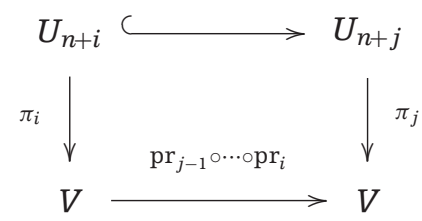

and it follows immediately from (.9) that

$$
V_{\beta_{k}}=\pi_{j_{k}}\left(U_{n+i_{k}}\right) .
$$


Since $\operatorname{dim} \operatorname{Ker}\left(\pi_{j_{k}}\right)=j_{k}-1$ and hence $\ell_{k}=i_{k}+\operatorname{dim} \operatorname{Ker}\left(\pi_{j_{k}}\right)$, we have an embedding of projective varieties:

$$
\begin{aligned}
\zeta_{\beta_{k}}: \mathrm{Gr}_{i_{k}}\left(V_{\beta_{k}}\right) \longleftrightarrow \mathrm{Gr}_{\ell_{k}}\left(U_{n+i_{k}}\right), \\
Z \longmapsto \pi_{j_{k}}^{-1}(Z) .
\end{aligned}
$$

This induces an embedding

$$
\zeta=\zeta_{n}:=\prod_{k=1}^{N} \zeta_{\beta_{k}}: \prod_{k=1}^{N} \mathrm{Gr}_{i_{k}}\left(V_{\beta_{k}}\right) \longleftrightarrow \prod_{k=1}^{N} \mathrm{Gr}_{\ell_{k}}\left(U_{n+i_{k}}\right)
$$

In view of (.10), the variety $R_{n}$ is contained in $\prod_{k=1}^{N} G_{i_{k}}\left(V_{\beta_{k}}\right)$ and we claim that the image $\zeta\left(R_{n}\right)$ is canonically isomorphic to the variety $B_{n}$ (see Definition A.3). Indeed let $U=\left(U_{\beta_{k}}\right)_{k=1}^{N}=\zeta\left(\left(Z_{\beta_{k}}\right)_{k=1}^{N}\right) \in \zeta\left(R_{n}\right)$, that is, $U_{\beta_{k}}:=\pi_{j_{k}}^{-1}\left(Z_{\beta_{k}}\right)$. We decorate the collection $U=\left(U_{\beta_{k}}\right)_{k=1}^{N}$ with the subspaces $U_{\beta_{-t}}:=F_{t}$, for $0 \leq t \leq 2 n$. The decorated collection $\tilde{U}=$ $\left(U_{\beta_{k}}\right)_{k=-2 n}^{N}$ satisfies (.16), (.17), (.18), and (.19), that is, $\tilde{U} \in B_{n}$. Indeed, (.16) is clearly satisfied, (.17) follows from (.10) and (.18) follows from (.11). Since $Z_{\beta_{k}} \subset V_{\beta_{k}}=\pi_{j_{k}}\left(U_{n+i_{k}}\right)$, there exists $U \subset U_{n+i_{k}}$ such that $Z_{\beta_{k}}=\pi_{j_{k}}(U)$; in view of (.12), $\operatorname{pr}_{j_{k}}\left(Z_{\beta_{k}}\right) \subset Z_{\beta_{k}^{+}}$and we have

$$
\pi_{j_{k}+1}^{-1}\left(Z_{\beta_{k}^{+}}\right) \supset \pi_{j_{k}+1}^{-1} \operatorname{pr}_{j_{k}}\left(Z_{k}\right)=\pi_{j_{k}+1}^{-1} \pi_{j_{k}+1}(U) \supseteq U=\pi_{j_{k}}^{-1}\left(Z_{k}\right)
$$

and hence $\tilde{U}$ satisfies (.19).

On the other hand, let $\left(U_{\beta_{k}}\right)_{k=-2 n}^{N}$ be a point of $B_{n}$. We define $Z_{\beta_{k}}:=\pi_{j_{k}}\left(U_{\beta_{k}}\right)(k=$ $1,2, \ldots, N)$ and we check that $\left(Z_{\beta_{k}}\right)_{k=1}^{N} \in R_{n}$, that is, it satisfies (.10), (.11), and (.12). In view of (.24), $Z_{\beta_{k}} \in V_{\beta_{k}}$. Since $U_{\beta_{k}^{-}} \subset U_{\beta_{k}}$ we have Ker $\pi_{j_{k}}=F_{j_{k}-1} \subseteq U_{\beta_{k}}$ and hence $\operatorname{dim} Z_{\beta_{k}}=$ $\ell_{k}-\left(j_{k}-1\right)=i_{k}$. It follows that $Z_{\beta_{k}} \in \mathrm{Gr}_{i_{k}}\left(V_{\beta_{k}}\right)$ and (.10) is satisfied. Since $U_{\beta_{k}^{-}} \subset U_{\beta_{k}}(.11)$ follows. To prove (.12), we note that

$$
\operatorname{pr}_{j_{k}}\left(Z_{\beta_{k}}\right)=\operatorname{pr}_{j_{k}} \circ \pi_{j_{k}}\left(U_{\beta_{k}}\right)=\pi_{j_{k+1}}\left(U_{\beta_{k}}\right) \subseteq \pi_{j_{k+1}}\left(U_{\beta_{k}^{+}}\right)=Z_{\beta_{k}^{+}}
$$

and the claim is proved.

In view of Proposition A.4, the map $\psi_{n}:=\theta_{n}^{-1} \circ \zeta_{n}: R_{n} \rightarrow \mathrm{BS}_{n}$ is hence an isomorphism of projective varieties. It remains to check that the diagram (.23) commutes. Using (.6), we obtain

$$
\rho_{n} \circ \psi_{n}\left(\left(Z_{\beta_{k}}\right)_{k=1}^{N}\right)=\left(\pi_{j_{k}}^{-1}\left(Z_{\alpha_{k, k}}\right)\right)_{k=1}^{n} .
$$


On the other hand, $p_{n}\left(\left(Z_{\beta_{k}}\right)_{k=1}^{N}\right)=\left(Z_{\alpha_{k, k}}\right)_{k=1}^{n}$, and

$$
\zeta \circ p_{n}\left(\left(Z_{\beta_{k}}\right)_{k=1}^{N}\right)=\left(\pi_{j_{k}}^{-1}\left(Z_{\alpha_{k, k}}\right)\right)_{k=1}^{n}
$$

as desired.

\section{References}

[1] Assem, I., D. Simson, and A. Skowronski, Elements of the Representation Theory of Associative Algebras. Vol. 1. Techniques of Representation Theory. London Mathematical Society Student Texts 65. Cambridge: Cambridge University Press, 2006.

[2] Cerulli Irelli, G., E. Feigin, and M. Reineke. "Quiver Grassmannians and degenerate flag varieties." Algebra \& Number Theory 6, no. 1 (2012): 165-94.

[3] Cerulli Irelli, G., E. Feigin, and M. Reineke. "Degenerate flag varieties: moment graphs and Schröder numbers." Journal of Algebraic Combinatorics 38, no. 1 (2013).

[4] Cerulli Irelli, G., E. Feigin, and M. Reineke, "Desingularization of quiver Grassmannians for Dynkin quivers." Advances in Mathematics 245 (2013): 182-207.

[5] Demazure, M. "Désingularisation des variétés de Schubert généralisées." Annales Scientifiques de l'École Normale Supérieure 7 (1974): 53-88.

[6] Deodhar, V. "On some geometric aspects of Bruhat orderings II. The parabolic analogue of Kazhdan-Lusztig polynomials." Journal of Algebra 111, no. 2 (1987): 483-506.

[7] Feigin, E. "Degenerate flag varieties and the median Genocchi numbers." Mathematical Research Letters 18, no. 6 (2011): 1-16.

[8] Feigin, E. “ $\mathbb{G}_{a}^{M}$ degeneration of flag varieties." Selecta Mathematica 18, no. 3 (2012): 513-37.

[9] Feigin, E. and M. Finkelberg. "Degenerate flag varieties of type A: Frobenius splitting and BW theorem." Mathematische Zeitschrift 275, no. 1-2 (2013): 55-77.

[10] Feigin, E., G. Fourier, and P. Littelmann. "PBW filtration and bases for irreducible modules in type $A_{n} . "$ Transformation Groups 16, no. 1 (2011): 71-89.

[11] Feigin, E., G. Fourier, and P. Littelmann. "PBW filtration and bases for symplectic Lie algebras." International Mathematics Research Notices 24 (2011): 5760-84.

[12] Feigin, E., G. Fourier, and P. Littelmann. "Favourable modules: filtrations, polytopes, Newton-Okounkov bodies and flat degenerations." (2013): preprint. arXiv: 1306.1292.

[13] Feigin, E., M. Finkelberg, and P. Littelmann. "Symplectic degenerate flag varieties." Canadian Journal of Mathematics. http://cms.math.ca/10.4153/CJM-2013-038-6.

[14] Fulton, W. Young Tableaux. London Mathematical Society Student Texts 35. Cambridge: Cambridge University Press, 1997.

[15] Hansen, H. C. “On cycles in flag manifolds." Mathematica Scandinavica 33 (1973): 269-74.

[16] Lakshmibai, V. and K. N. Raghavan. Standard Monomial Theory. Invariant Theoretic Approach. Encyclopaedia of Mathematical Sciences 137. Invariant Theory and Algebraic Transformation Groups 8. Berlin: Springer, 2008. 